\title{
Object Detection by Estimation of the Spectral Attenuation Coefficient in the Submerged Medium
}

\author{
Tajbia Karim ${ }^{1}$, Ebad Zahir $^{2}$ \\ ${ }^{1,2}$ Department of EEE, American International University-Bangladesh
}

\begin{abstract}
Underwater object detection has a considerable impact in the field of ocean technology. This paper presents a techniqueto detect a submerged object based on the medium's spectral attenuation coefficient by applying Beer-Lambert Law. The value of this coefficient is a function of the medium's composition, depth of the object,and wavelength of incident light. Experiments were performed in a swimming pool using objects of different colors at different depths to validate the mathematical model for object detection.
\end{abstract}

Keywords: Attenuation Coefficient, Beer-Lambert Law, Color Attenuation, Object Detection.

\section{Introduction}

Detecting objects underwater has always presented a number of possible applications especially in cases such asavoiding underwater barriers, repairing underwater structures, and implementing marine technology. SONAR imaging is a popular method for detecting underwater objects. Sound was preferred over color in underwater mediums because of its lower attenuation with respect to distance. Single sonar imagery has been used largely forunderwater object detection [1].

Multi-sensor detection technology using three different sonar images containing varying frequency and bandwidth characteristics has been performed[2],[3]. However, color can be an essential attribute on object detection or tracking technology because of minimalism, or sturdiness, especially where a very specific object needs to be detected that has similar shaped objects (manmade or natural) in its surroundings. An example can be the detection of naval mines. Lately, numerous underwater vehicles have utilized color based system as major sensing method [4],[5] for improved resolution and cost efficiency. A color matching method based on the modeling of the color modification by the water has already been used[6] wherean underwater object detection technique based on principle component analysis has been proposed. The underwater object detection involved the application of Beer-Lambert Law and later on with the help of principle component analysis, used numbers of submerged manmade object's sample todetect the object's color.

G.L. Foresti and S. Gentili also represented vision based underwater object detection. A color compensation process has been introduced in their work. They have applied artificial neural networks to classify in real-time the pixels of the input image into different classes. Geometric reasoning is used to improve the accuracy of desired object detection. [7]

In this paper, underwater objects have been detected deriving water's spectral attenuation coefficient. It is a detection method for objects that are already known. By applying Beer-Lambert Law on only two samples of experimental data, the medium's (water) spectral coefficient has been calculated. It differs majorly depending on the water's composition, dwelling materials like phytoplankton, etc. It has also been shown in this paper that knowledge of spectral attenuation coefficient's value as well as the initial color information of the object leads to predicting the color of that object at different depths. The feasible depth varies depending on the water constituent, especially, suspended materials in the water body. Methodology of the detection has been explained in section III. Section IV discusses the experimental results and success rates based on the submerged object's color.

\section{Methodology}

While electromagnetic wave travels through water medium, it gets attenuated by converting into other forms of energies. The amount of attenuation varies with respect to wavelength. For the visible spectrum, longer wavelengths are absorbed before shorter wavelengths. The depth dependence attenuation of light can be described by equation (1) below [8]:

$$
\mathrm{E}_{\mathrm{d}}(\mathrm{z}, \lambda)=\mathrm{E}_{\mathrm{d}}(0, \lambda) \exp \left[-\int_{0}^{\mathrm{z}} \mathrm{K}_{\mathrm{d}}\left(\mathrm{z}^{\prime}, \lambda\right) \partial \mathrm{z}^{\prime}\right]
$$

Here, $K_{d}\left(z^{\prime}, \lambda\right)$ is the coefficient that defines the pattern of attenuation of every wavelength of spectrum. It can be defined as spectral attenuation coefficient and $\mathrm{E}_{\mathrm{d}}$ as down-welling irradiance dependant on wavelength. 
From equation (1),

$$
\mathrm{K}_{\mathrm{d}}(\mathrm{z}, \lambda)=-\frac{\partial \ln \mathrm{E}_{\mathrm{d}}(\mathrm{z}, \lambda)}{\partial \mathrm{z}}=-\frac{1}{\mathrm{E}_{\mathrm{d}}(\mathrm{z}, \lambda)} \frac{\partial \mathrm{E}_{\mathrm{d}}(\mathrm{z}, \lambda)}{\partial \mathrm{z}}
$$

The mean $K_{d}(z, \lambda)$ from depth 0 to $z$ distance would yield as:

$$
\overline{\mathrm{K}_{\mathrm{d}}}(\mathrm{z}, \lambda)=\frac{1}{\mathrm{z}} \int_{0}^{\mathrm{z}} \mathrm{K}_{\mathrm{d}}\left(\mathrm{z}^{\prime}, \lambda\right) \partial \mathrm{z}^{\prime}
$$

According to expression (2), equation (1) can be modified as:

$$
\mathrm{E}_{\mathrm{d}}(\mathrm{z}, \lambda)=\mathrm{E}_{\mathrm{d}}(0, \lambda) \exp \left[-\overline{\mathrm{K}_{\mathrm{d}}}\left(\mathrm{z}^{\prime}, \lambda\right) \mathrm{z}\right]
$$

According to the findings of Howard R. Gordon, the value of $K_{d}(z, \lambda)$ shows very slow variation with depth[9]. So, for simplified and faster calculations, consideration of $\overline{K_{d}}(z, \lambda)$ on only wavelength is justified.

Thus, a clear dependency of the variation of different wavelengths on the value of $\overline{K_{d}}$ can be utilized to find an algorithm of detecting all the probable value of $E_{d}(z, \lambda)$ which would occur at different oceanic depths. The simplified form of algorithm can be described using the following steps-

i. Accumulation of irradiance information from the surface of the object. Let's consider this information to be $\mathrm{E}_{\mathrm{d}}(0, \lambda)$

ii. Collection of irradiance information submerging the object at a fixed depth $\mathrm{z}$ under the water surface.

iii. With the known parameters from steps $\mathrm{i}$ and ii, value of $\overline{\mathrm{K}_{\mathrm{d}}}$ can be determined for different wavelengths. In this paper, $\overline{\mathrm{K}_{\mathrm{d}}}$ values have been determined for Red, Green and Blue channels for each object (as every object's color can be defined with RGB color space).

iv. Applying the values of $\overline{K_{d}}$ values in equation 3 , color information of the object at different depth levelE $E_{d}(z, \lambda)$ can be determined by varying the value of $z$.

v. The obtained colors at step ivis now considered as the compatible color of the object. The list of this compatible color indicates pattern of color changes of an object.

vi. With knowledge of the compatible colors, it is possible to identify the presence of desired objects in an underwater environment. For this purpose, the HSV (Hue, Saturation and Values)color information of every compatible color has been collected and an automatic matching process has been run using the suggested algorithm. The tolerance levels of Hue, Saturation and Values have been tested and verified for best detection.

\section{Experimental Results}

To verify the accuracy of the previously described algorithm, 3 different colored objects (blue, green, red) have been detected under different depths underwater experimentally.

\section{Detection of a Blue Object}

According to the algorithm, the irradiance information has been accumulated from the following two samples of the blue object-

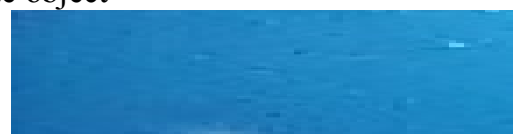

(a)

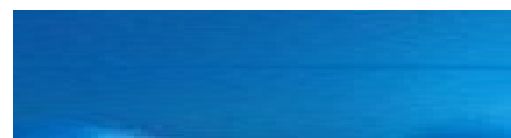

(b)

Fig. 1: Samples considered for blue object. (a)Un-submerged sample(depth=0) and (b) Submerged sample (depth=1 foot).

Applying the equation (2), the value of spectral attenuation coefficient of red, green and blue channels are respectively, $\mathrm{K}_{\mathrm{r}}=0.0387$ per centimeter, $\mathrm{K}_{\mathrm{g}}=0.0046$ per centimeter and $\mathrm{K}_{\mathrm{b}}=0.0011$ per centimeter. Using these coefficient values in equation (3), and varying $\mathrm{z}$ from 0 to 304.8 centimeter(10 feet), the compatible color for this object can be predicted as follows-

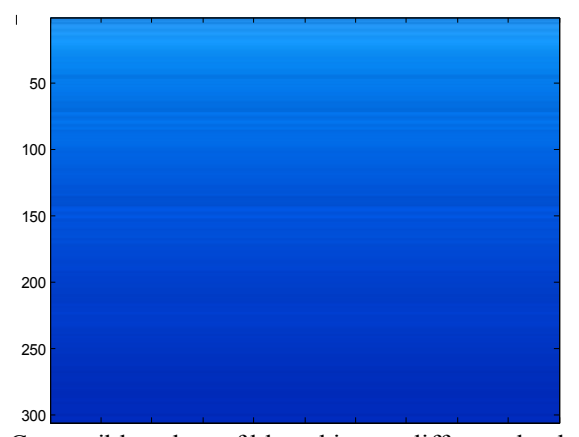

Fig. 2: Compatible colors of blue object at different depths under water

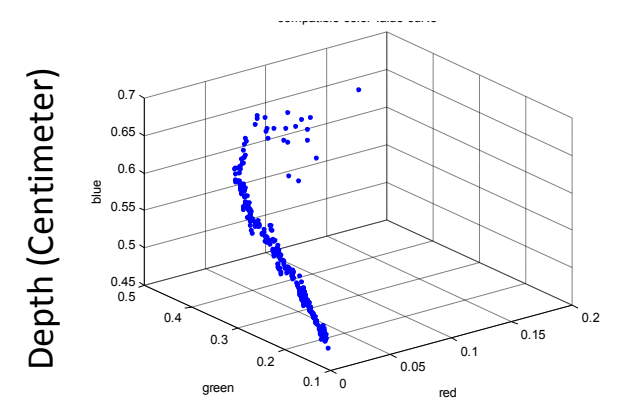

Fig. 3: The RGB coordinates values representation of compatible colors 
In fig.3,the close proximity of represented values towards green and blue coordinates is comprehensible from the compatible color list. Using the pattern of color variationof fig.2, the object has been detected at various underwater environment images at various depths. To detect the object, all the pixels of compatible color list have been read from fig. 2 and then an automated matching process of those pixels has been performed using underwater environment images to locate the existence of the object. The existence (match) is shown to be marked with white pixels and non-match is markedwith black pixels.

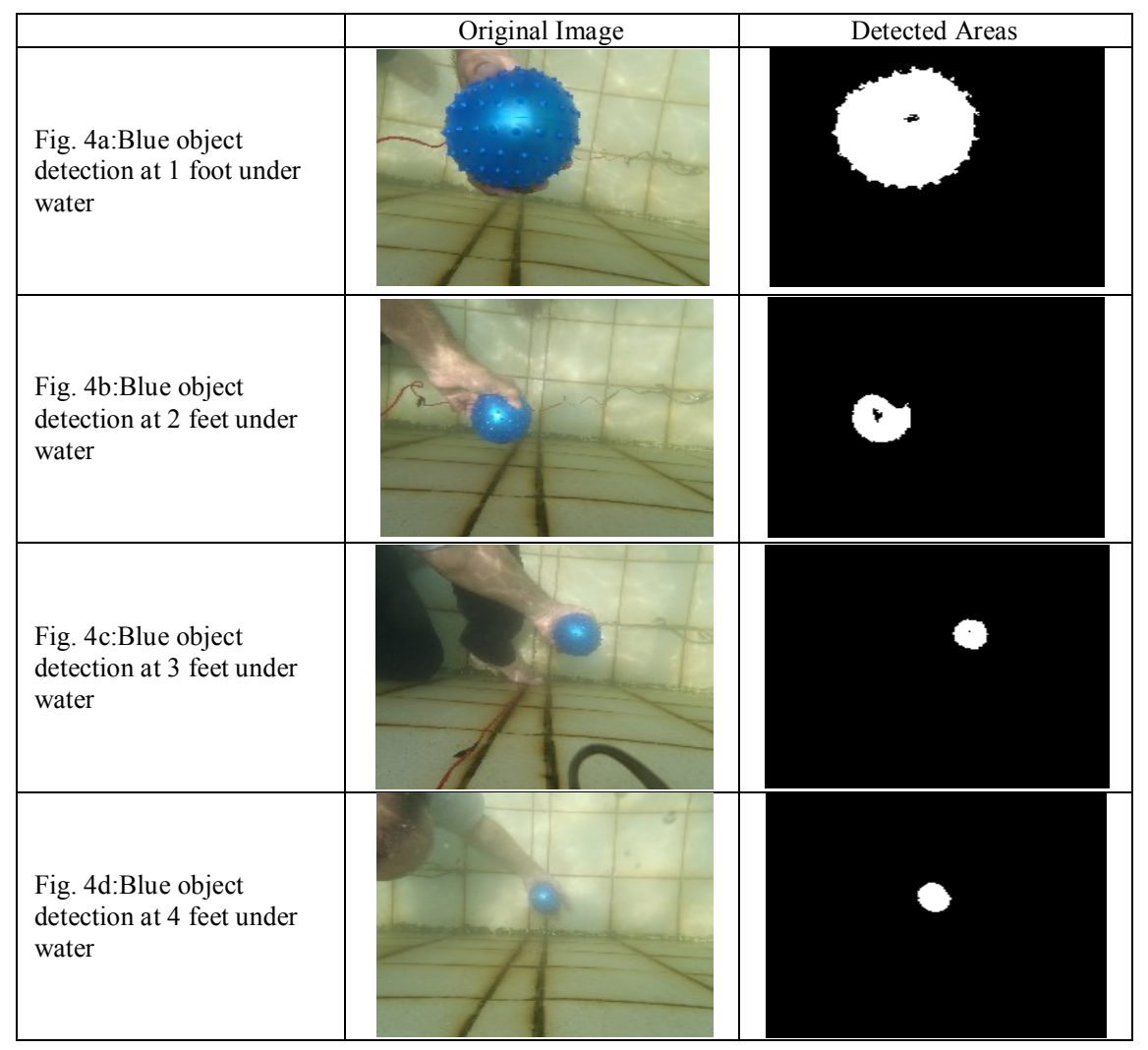

For detection of the blue object at depth 1 foot, different tolerance levels for Hue, Saturation and Values have been used and for the application of tolerance as Hue $=0.1$,Saturation $=0.40$ and Value $=0.4$, the ball was detected most precisely with the least number of noise and maximum detected area of the object. It was able to pick a total of 216602 pixels among 249309. The same object could be detected most perfectly at 2 feet depth when tolerance levels of Hue $=0.11$ Saturation $=0.55$ and Value $=0.55$ were used. The increased tolerance value of Saturation and Value indicate that the object has shifted from its intensity and lightness/darkness information of the compatible colors. Total pixel 34555 pixels could be detected among 44754 with this tolerance level.At 3 feet underwater best result was achieved using tolerance of Hue, Saturation and Value levels respectively as- $0.11,0.55$ and 0.55 i.e., the same as 2 feet depth detection. It is an indication that at certain depth, being at almost same amount of source light (in this casethe sun),the object appears to be of same compatible color information. Total pixels detected were 12479 among 27468 pixels. At 4 feet depth, HSV tolerance considered for best detection is $0.19,0.9$ and 0.9 respectively. That means intensity and lightness/darkness information has further shifted from previous level. The lower amount of received source light and unclear water because of the impurity can be the reason behind this change. Total 11525 pixels were among 12978.Using interpolation, equations of appropriate Hue, Saturation and Value, levels have been derived for the blue object as follows-

$$
\begin{aligned}
& \text { Hue }=0.015 \mathrm{x}^{3}-0.095 \mathrm{x}^{2}+0.19 \mathrm{x}-0.01 \\
& \text { Saturation }=0.0833 \mathrm{x}^{3}-0.575 \mathrm{x}^{2}+1.2917 \mathrm{x}-0.4 \\
& \text { Value }=0.0833 \mathrm{x}^{3}-0.575 \mathrm{x}^{2}+1.2917 \mathrm{x}-0.4
\end{aligned}
$$

In these equations and further, $\mathrm{x}$ represents the amount of underwater depth.

\section{Detection of a Red Object}

Similar to the blue object, the following samples have been used for calculation of attenuation coefficients of the red object- 


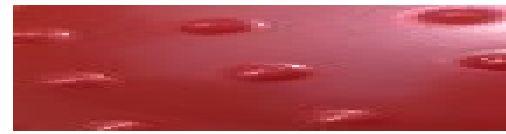

(a)

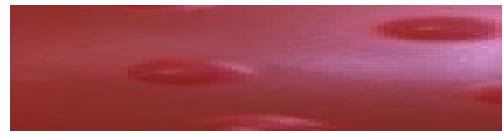

(b)

Fig 5:Samples considered for red object. (a)Un-submerged sample (depth=0) and (b) Submerged sample (depth=1 foot).

The derived attenuation coefficient values of red, green and blue channels are respectively- $\mathrm{K}_{\mathrm{r}}=0.0028 \mathrm{per} \mathrm{cm}$, $\mathrm{K}_{\mathrm{g}}=-3.4429 \mathrm{e}-04$ per $\mathrm{cm}$ and $\mathrm{K}_{\mathrm{b}}=-0.0022$ per $\mathrm{cm}$.
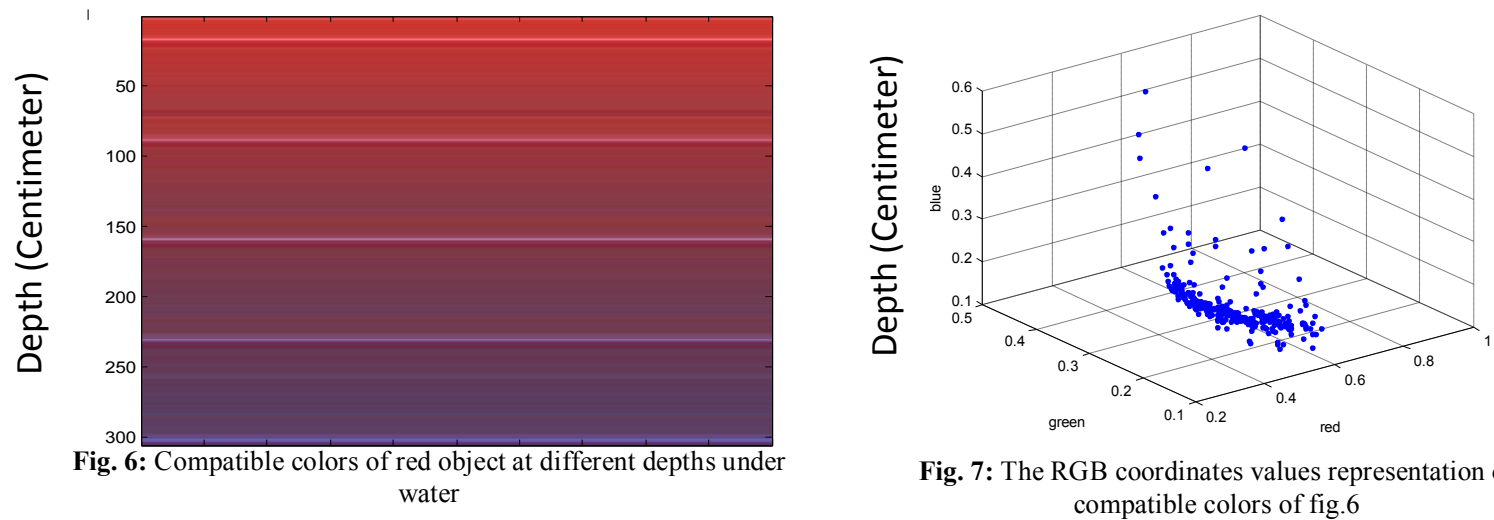

Fig. 7: The RGB coordinates values representation of compatible colors of fig. 6

Fig. 7 describes the existence of the pixel points at mostly at the middle of the red channel i.e., around 0.5.This is also visibly clear from fig.6.

\begin{tabular}{|l|l|l|}
\hline & Original Image & Detected Areas \\
\hline $\begin{array}{l}\text { Fig. 8a: Red object } \\
\text { detection at 1 foot under } \\
\text { water }\end{array}$ & & \\
\hline $\begin{array}{l}\text { Fig. 8b: Red object } \\
\text { detection at 2 feet under } \\
\text { water }\end{array}$ & & \\
\hline $\begin{array}{l}\text { Fig. 8c: Red object } \\
\text { detection at 3 feet under } \\
\text { water }\end{array}$ & & \\
\hline $\begin{array}{l}\text { Fig. 8d: Red object } \\
\text { detection at 4 feet under } \\
\text { water }\end{array}$ & & \\
\hline
\end{tabular}

Detection of the red colored object wasnot as accurate as the blue one. One of the most likelyreasons is that unlike blue, red cannot travel through the water for a great distance. Being a longer wavelength, it gets absorbed after traveling a short distance. Another reason is the existence of iron in the pool water where the experiment was conducted. The second reason is not going to affect as much in sea water as there isn't presence of as much iron mineral.

From the experiment, HSV tolerance considered for best detection at 1 foot depth were $0.2,0.3$ and 0.3 respectively. It could detect 31429 pixels among 124810 . Though the number of detected pixels wasn't very 
high but it didn't pick any noise.For 2 feet depth, image appropriate HSV tolerance level was found 0.3,0.3 and 0.3. Only tolerance of Hue had to be increased than previous stage. It can be realized that the object's wavelength certainly appears to be shifted from compatible color while keeping the intensity and darkness information the same. Total detected pixels were 10619 among 97126. It was also a noise free detection. At 3 feet depth, HSV tolerance considered for best detection was $0.28,0.45$ and 0.45 . Higher allowance of Saturation and Value tolerance means the intensity information has been deviated further. Total detected pixels at this depth were 16817 among 13138. However, the red object could be detected at 4 feet depth using higher tolerance of Hue information. This time HSV tolerance considered for best detection is $0.4,0.32$ and 0.32 and total detected pixels include a lot of noise. Almost one-eighth of the object pixels could be found.

Equations of appropriate Hue, Saturation and Value levels have been derived for the red object as follows-

$$
\begin{aligned}
& \text { Hue }=0.0433 \mathrm{x}^{3}-0.32 \mathrm{x}^{2}+0.7567 \mathrm{x}-0.28 \\
& \text { Saturation }=0.053 \ln (\mathrm{x})+0.3004 \\
& \text { Value }=0.053 \ln (\mathrm{x})+0.3004
\end{aligned}
$$

\section{Detection of a Green Object}

Samples considered for derivation of compatible colors are-

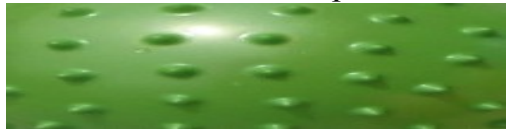

(a)

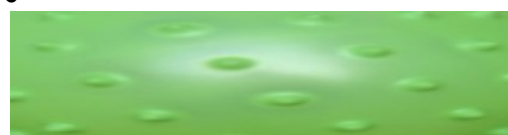

(b)

Fig.9:Samples considered for green object. (a)Un-submerged sample (depth=0) and (b) Submerged sample (depth=1 foot).

The attenuation coefficients calculated are $\mathrm{K}_{\mathrm{r}}=-0.0100$ per $\mathrm{cm}, \mathrm{K}_{\mathrm{g}}=-0.0096$ per $\mathrm{cm}$ and $\mathrm{K}_{\mathrm{b}}=-0.0186$ per $\mathrm{cm}$.

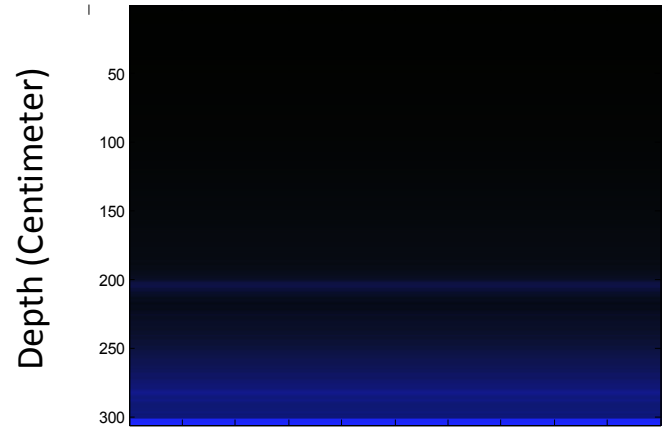

Fig. 10: Compatible colors of green object at different depths under water

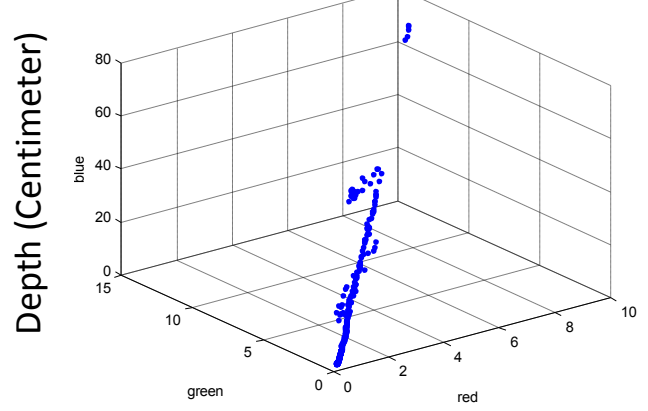

Fig 11: The RGB coordinate values representation of compatible colors of fig 10 .

The bluish compatible color from green colored object samples occur as blue and green colors wavelengths are very close. So while calculating the attenuation coefficients, the attained values lead the compatible colors toward blue. Though the compatible colors didn't seem much to be related to the green object, but detection of green object could be easily performed using the compatible color list. But detection of green and blue objects can be ambiguous. The experiment shows that the compatible colors of green object can easily detect a blue object of similar intensity. But the compatible colors of blue object hardly detect any green object. Use of samples and underwater environment image with very high number of pixels and lower tolerance level can reduce the ambiguity to a great extent.

Detection of green object at different depths using the compatible colors for fig. 10 has been shown below:

\begin{tabular}{|l|l|l|}
\hline & Original Image & \\
\hline $\begin{array}{l}\text { Fig. 12a: Green object } \\
\text { detection at 1 foot under } \\
\text { water }\end{array}$
\end{tabular}




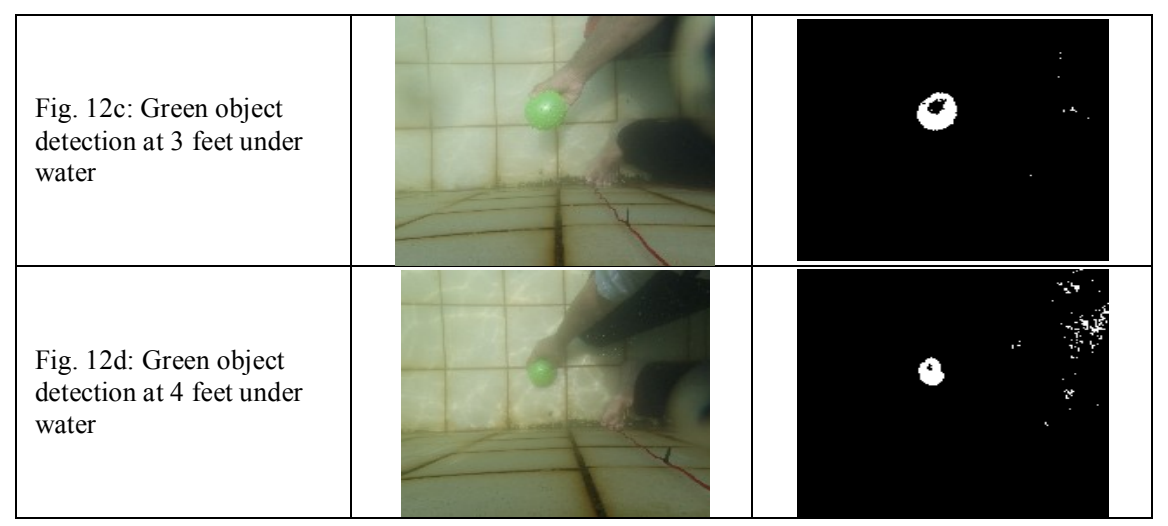

For the green object, the tolerance levels of Hue, Saturation and Value didnot vary noticeably with depth. At 1 foot depth, HSV tolerance considered for best detection was $0.45,0.45$ and 0.45 respectively which would lead to detect total pixels 96171 among 139798. As noise, mostly very dark colors (tends to black), were picked but it wasn't at a high amount.At 2 feet depth, the green object was best found using tolerance of Hue 0.44 , Saturation 0.52 and Value 0.52 . The allowance on intensity information has been increased from the previous stage. Total detected pixels were 40862 among 50878. Noise percentage was again negligible.For 3 feet depth, the best result could be achieved for HSV tolerance of $0.4405,0.55$ and 0.55 respectively which is very close to levels of 2 feet. Total detected pixels are 18218 among 2271.Again, the green object could be detected with low noise pixels. HSV tolerance considered for best detection at 4 feet depth is $0.44,0.55$ and 0.55 respectively. Total detected pixels were 18215 among 10470 . Noise pixels were recorded to be more than previous stages. But lower noise pixel density separates the object from the environment.

Equations of appropriate Hue, Saturation and Value levels have been derived for green object as follows-

$$
\begin{aligned}
& \text { Hue }=-0.0017 \mathrm{x}^{3}+0.0147 \mathrm{x}^{2}-0.0421 \mathrm{x}+0.479 \\
& \text { Saturation }=0.0167 \mathrm{x}^{3}-0.15 \mathrm{x}^{2}+0.4333 \mathrm{x}+0.15 \\
& \text { Value }=0.0167 \mathrm{x}^{3}-0.15 \mathrm{x}^{2}+0.4333 \mathrm{x}+0.15
\end{aligned}
$$

\begin{tabular}{|c|l|c|c|c|c|}
\hline \multicolumn{3}{|c|}{ Table1: The appropriate tolerance levels of different colored objects at } \\
different depths \\
\hline \multirow{3}{*}{ Object Color } & \multicolumn{3}{|c|}{$\begin{array}{c}\text { Tolerance level considered for Hue, Saturation } \\
\text { and Value respectively at different depths }\end{array}$} \\
\cline { 3 - 6 } & 1 foot & 2 feet & 3 feet & 4 feet \\
\hline \multirow{3}{*}{ Blue } & Hue & 0.1000 & 0.1100 & 0.1100 & 0.1900 \\
\cline { 2 - 6 } & Saturation & 0.4000 & 0.5500 & 0.5500 & 0.9000 \\
\cline { 2 - 6 } & Value & 0.4000 & 0.5500 & 0.5500 & 0.9000 \\
\hline \multirow{3}{*}{ Red } & Hue & 0.2000 & 0.3000 & 0.2800 & 0.4000 \\
\cline { 2 - 3 } & Saturation & 0.3000 & 0.3000 & 0.4500 & 0.3200 \\
\cline { 2 - 6 } & Value & 0.3000 & 0.3000 & 0.4500 & 0.3200 \\
\hline \multirow{3}{*}{ Green } & Hue & 0.4500 & 0.4405 & 0.4405 & 0.4400 \\
\cline { 2 - 6 } & Saturation & 0.4500 & 0.5500 & 0.5500 & 0.5500 \\
\cline { 2 - 6 } & Value & 0.4500 & 0.5500 & 0.5500 & 0.5500 \\
\hline
\end{tabular}

For verification of the process, an underwater image was taken at 2 feet depth where both red and green colored objects were present. According to the previous discussion, by reading the compatible colors of green, and running the matching process to this image, would detect the green object. The result is as follows-
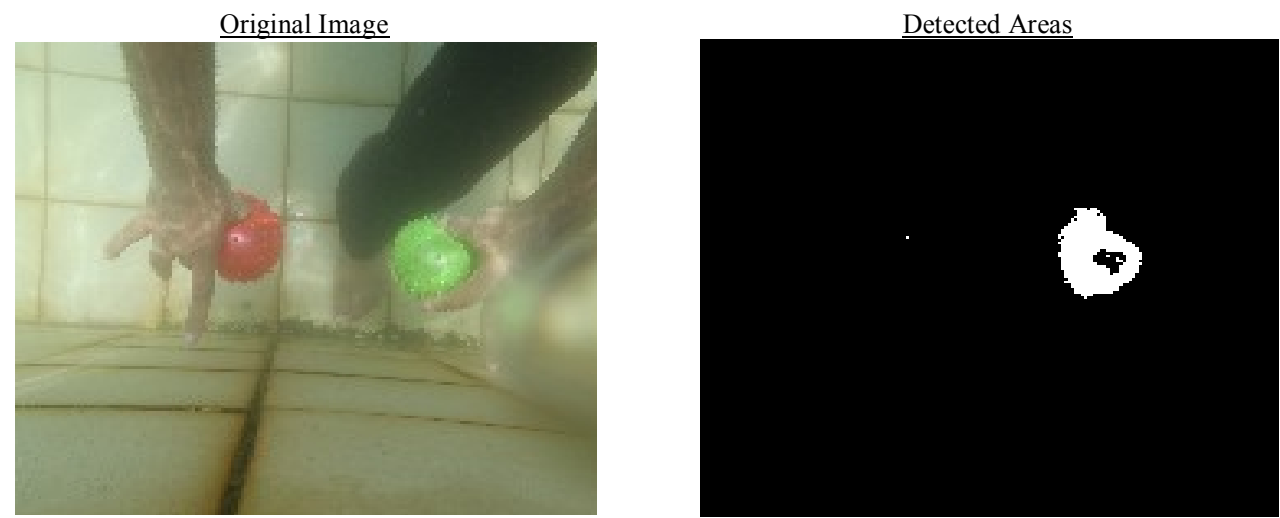

Fig 13: Detection of green object with the presence of a red object 
Figure 13shows that green object was detected almost perfectly. The HSV tolerance level used were $0.4405,0.55$ and 0.55 following theequation (10), (11) and (12).

For further verification, compatible colors of red object were read and the matching process was repeated on the same image which led to one-eighth portion detection of the red object:
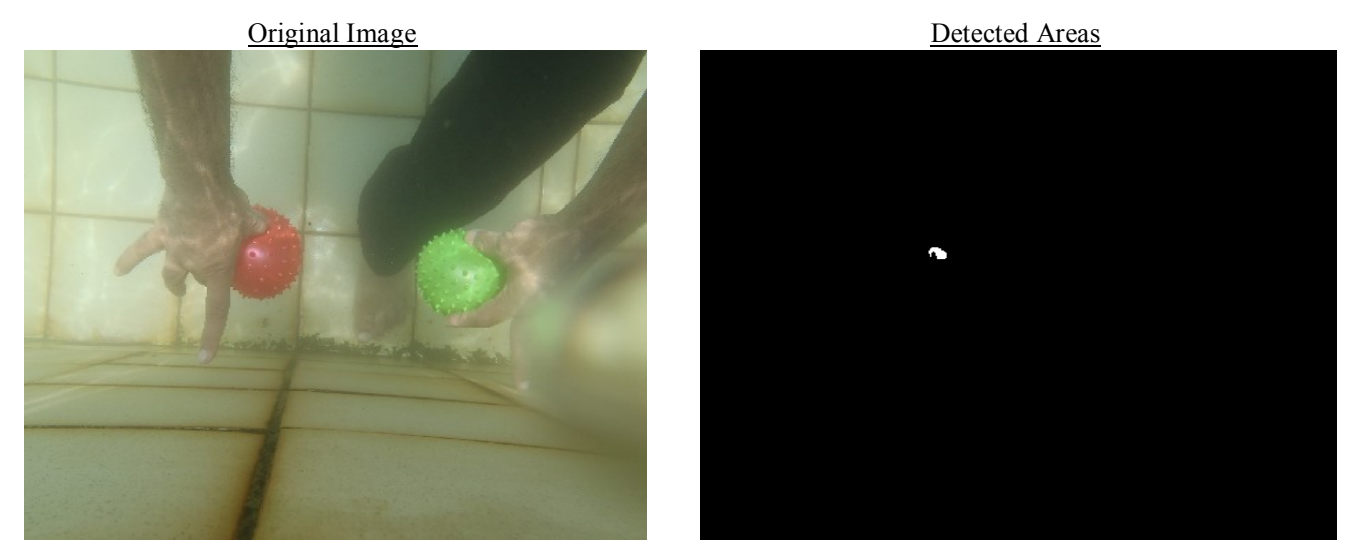

Fig 14: Detection of green object with the presence of a red object

\section{Conclusion}

Submerged objects of three major colors- red, green and blue have been detected with the estimation of water medium's attenuation coefficient values. From the experiment, the maximum portion has been detected for the blue object and the least for the red object. One of the main reasons is the absorption of longer wavelength before shorter wavelength while traveling through the medium. It has also caused the minimum variation of Hue tolerance level for the blue object compared to the other colored objects. The increased deviation of Saturation and Value with depth is also noticeable for all colored objects. The detection process can be further utilized if the color feature was the main attribute used for identification. The identification range will be a function of the medium's attenuation coefficient's value which is expected to vary in different waterbodies.

\section{References}

[1]. J. D. Tucker, Coherence-based underwater target detection for side-scan sonarimagery, Master's Thesis, Colorado State University, 2009.

[2]. T. Aridgides and M. F. Fernandez, Automated target classification in highresolution dual frequency sonar imagery, Proc. SPIE, , April 2007 ,vol. 6553,pp. 1-12.

[3]. T. Aridgides and M. Fernandez, Enhanced ATR algorithm for high resolutionmulti-band sonar imagery, Proc. SPIE, March 2008,vol. 6953, pp. 0-1.

[4]. G. Dudek et al.,A visually guided swimming robot, Proc. IEEE International Conference on Intelligent Robots and Systems, 2005, pp.3604-3609.

[5]. J. Sattar, P. Giguere, G. Dudek, and C. Prahacs, A visual servoing system for an aquatic swimming robot, Proc. IEEE International Conference on Intelligent Robots and Systems, 2005, pp. 1483-1488.

[6]. StéphaneBazeille, Isabelle Quidu and Luc Jaulin, Color-based underwater object recognition using water light attenuation,Intelligent Service Robotics, , April 2012,Volume 5, Issue 2, pp 109-118.

[7]. G.L. Foresti and S. Gentili,avison based system for object detection in underwater images, International Journal of Pattern Recognition and Artificial Intelligence, Vol. 14, No. 2 (2000) pp. 167-188

[8]. J. Ahlen, Color correction of underwater images using spectral data, Thesis, Uppsala University, Centre for Image Analysis, 2005.

[9]. Gordon, H., Theoretical aspects of hydrologic optics. Limnology and Oceanography. 34(8), 1989, $1389-1409$. 